\title{
Relación entre funciones ejecutivas y conciencia fonológica en autismo: un estudio de caso
}

\section{Relationship between executive functions and phonological awareness in autism: a case study}

\author{
Yolanda Ares Comba \\ Facultad de Ciencias de la Educación. Universidade da Coruña
}

\begin{abstract}
Resumen
En este trabajo participó un niño de 7 años diagnosticado de trastorno del espectro autista (TEA) con el que se llevó a cabo un entrenamiento en funciones ejecutivas con el fin de comprobar si se producía una mejora en la memoria de trabajo, la velocidad de procesamiento, la conciencia fonológica y la competencia lectora inicial. El estudio se llevó a cabo a lo largo de tres fases: evaluación previa a la intervención, fase de entrenamiento en funciones ejecutivas y evaluación posterior a la intervención. Los datos resultantes del entrenamiento en funciones ejecutivas, mostraron una mejora en las principales variables objeto de estudio.

Palabras clave: conciencia fonológica, TEA, funciones ejecutivas, memoria de trabajo, velocidad de procesamiento, competencia lectora inicial.
\end{abstract}

\begin{abstract}
In this study participated a 7 year old boy diagnosed with autism spectrum disorder (ASD) with which it conducted a training executive functions in order to check whether improved working memory was produced, the processing speed, phonological awareness and early reading skills. The study was conducted over three phases: pre-intervention assessment, phase of training in executive functions and post-intervention assessment. The data resulting from training executive, showed an improvement in the main variables under study.

Keywords: phonological awareness, ASD, executive functions, working memory, processing speed, initial reading skills
\end{abstract}

La conciencia fonológica ha sido considerada en las últimas décadas como una de las mejores evidencias del aprendizaje lector. Si bien existe abundante literatura que nos muestra la relación entre la conciencia fonológica y la adquisición de la lectura y la escritura, la interpretación de esta relación es tema central de controversias.

Bravo Valdivieso (2002), asegura que el aprendizaje inicial de la lectura estaría determinado principalmente por el desarrollo fonológico. Este compone el nivel de desarrollo más próximo para aprender a identificar los componentes del lenguaje escrito. La relación entre el desarrollo fonológico y el aprendizaje lector puede darse de tres maneras: como un desarrollo previo y predictivo, que contribuye a determinar un umbral cognitivo sobre el cual se inicia la decodificación; como una relación causal, siendo el desarrollo fonológico previo una condición dinámica que determina el aprendizaje posterior de la lectura, y como un proceso interactivo y recíproco con este aprendizaje, que tendría fases previas y fases posteriores, según lo determine la instrucción lectora. Esta situación implica que a partir de cierto umbral de sensibilidad fonológica a los componentes del lenguaje oral, los niños van desarrollando la conciencia fonológica del lenguaje escrito en la medida en que empiezan a decodificar las letras en una interacción con su enseñanza.

Heimann, Nelson, Tjus y Gillberg (1995) incluyeron una medida de la conciencia fonológica en su estudio sobre el efecto del uso de una forma interactiva e iniciada en el niño a través de un programa de ordenador (Alpha) para la enseñanza de la lectura y la escritura. En este trabajo participaron 11 niños con autismo, 9 niños con distintas dificultades y 10 niños con desarrollo típico cuyas edades oscilaban entre los 6 y los 13 años. Las puntuaciones medias en conciencia fonológica en los tres grupos de niños indicaron puntuaciones significativamente más bajas en los grupos de niños con autismo y con distintas dificultades en comparación con grupo de niños con desarrollo típico.

Siguiendo esta línea de investigación de las habilidades lingüísticas en TEA, Newman et al. (2007) llevaron a cabo un estudio en el que compararon las habilidades relacionadas con la lectura de los niños con TEA que presentaban hiperlexia (HPL), con niños de la misma edad con TEA sin HPL y con niños con desarrollo típico de lectura. Los resultados obtenidos mostraron que los niños con TEA e HPL realizaban mejor las tareas de lectura de una sola palabra y decodificación de pseudopalabras que los niños con TEA sin HPL.

Por su parte Gabig (2010) examinó la conciencia fonológica y la lectura de una sola palabra en un grupo de 14 niños con autismo y un grupo de 10 niños con desarrollo típico, de edades comprendidas entre los 5 y los 7 años. Los resultados mostraron que el grupo de niños con autismo puntuaba por debajo del promedio en las dos medidas de conciencia fonológica (tareas de omisión y mezcla de sonidos) en comparación con el grupo de niños con desarrollo típico.

En relación a las funciones ejecutivas, hace poco más de una década que los déficits ejecutivos en el autismo han comenzado a ser considerados importantes en el conjunto de la sintomatología autista, pasando de considerarlos como un déficit adicional a ser un claro objeto de estudio. En este sentido, el primer estudio que 
analizó las alteraciones de las funciones ejecutivas en personas con TEA, fue realizado por Rumsey (1985). En él participaron 9 personas adultas con TEA de edades comprendidas entre 18 y 39 años, sin discapacidad cognitiva asociada. Los resultados obtenidos mostraron diferencias significativas en cuanto al número de categorías completadas, el número de errores y el número de respuestas perseverativas entre el grupo de personas con TEA y el grupo control.

En esta misma línea, Ozonoff, Pennington y Rogers (1991) compararon un grupo de 23 personas diagnosticadas con TEA con un grupo control compuesto por 20 sujetos con dislexia, trastornos de aprendizaje, TDAH y retraso mental ligero. Los resultados mostraron diferencias significativas entre el grupo con TEA y los grupos control en la medida de flexibilidad.

Años más tarde, Towgood, Meuwese, Gilbert, Turner y Burgess (2009) exploraron en personas adultas los déficits cognitivos relacionados con el funcionamiento en tareas ejecutivas. En el estudio participaron 21 adultos diagnosticados con TEA de alto funcionamiento $\mathrm{y}$ un grupo control conformado por 22 participantes. Los resultados mostraron un conjunto limitado de déficits en las medidas de procesamiento y velocidad de la función ejecutiva en el grupo con TEA, la cual está encargada del inicio de respuesta, la inhibición y la memoria visual.

Tras todo lo expuesto y a pesar de la importancia que tiene la conciencia fonológica en la adquisición de la lectoescritura, es importante destacar el hecho de que se sabe poco sobre este aspecto en los niños con autismo, ya que son pocos los estudios que se centran en esta temática. Por este motivo, se llevó a cabo el presente trabajo con el objetivo de comprobar si un entrenamiento en funciones ejecutivas en un niño de 7 años de edad con TEA podría producir cambios positivos en la memoria de trabajo, la velocidad de procesamiento, así como en la conciencia fonológica y en la competencia lectora inicial. El estudio se llevó a cabo a lo largo de tres fases, una fase de evaluación previa a la intervención en la que se recogieron datos acerca de las cuatro variables objeto de estudio, una segunda fase de entrenamiento en funciones ejecutivas, y una tercera fase de evaluación con el fin de comprobar si se produjeran cambios en las variables analizadas tras la intervención. A continuación se describe de manera precisa cada una de las fases del trabajo, así como los resultados obtenidos.

\section{Método}

\section{Participantes}

En el estudio participó un niño de 7 años y 5 meses de edad diagnosticado de TEA. Que cursaba $2^{\circ}$ curso de Educación Primaria y que dos días por semana acudía a sesiones de entre 30-45 minutos, a la Asociación de Padres de Personas con Trastorno del Espectro Autista de La Coruña (ASPANAES).

A nivel curricular, el niño cuenta con adaptaciones curriculares en ambas lenguas (gallega y castellana). Acude al aula de Audición y Lenguaje cuatro días a la semana durante una hora para trabajar distintos aspectos de las habilidades lingüísticas.
A nivel verbal el niño se comunica de forma correcta, siendo capaz de establecer diálogos cortos y contestar a las preguntas que se le realizan.

A nivel lector, el niño identifica ya la mayoría de las letras y sus sonidos, por lo que la identificación de grafema-fonema está en un nivel medio-alto. Muestra lectura silabeada. Sin embargo, presentan dificultades a nivel de la escritura, pues la grafía no es adecuada.

Presenta deficiencias en la comunicación y en la interacción social en diversos contextos, por ejemplo en la reciprocidad socioemocional y en las conductas comunicativas no verbales, ya que apenas manifiesta contacto visual. También presenta dificultades para ajustar su comportamiento a los diversos contextos.

\section{Instrumentos}

- Prueba de Evaluación de Lenguaje Oral y Lenguaje Escrito (LolEva) (Fernández Amado, Mayor, Zubiauz, Tuñas, y Peralbo, 2006; Mayor et al., 2011). Evalúa conciencia fonológica y competencia lectora inicial - PEBL (Psychology Experiment Building Language) (Mueller y Piper, 2014). Programa de software de código abierto que incluye un conjunto de más de 50 pruebas psicológicas comunes como parte de su batería de pruebas. En este estudio se emplearon 2 de estas pruebas:

- Corsi Block-tapping Test o Prueba Corsi: evalúa memoria de trabajo

- Letter-Digit Substitution Task o Prueba Letter-Digit: evalúa velocidad de procesamiento

- Programa para la atención educativa al alumnado con TDAH (Artiles y Jiménez, 2006). Se utilizó como marco de referencia para diseñar las actividades de la fase de intervención

\section{Procedimiento}

El trabajo realizado se llevó a cabo en tres fases: evaluación previa a la intervención, entrenamiento en funciones ejecutivas, y evaluación una vez finalizada la intervención.

La fase de evaluación previa a la intervención se desarrolló a lo largo de dos sesiones en un total de hora y media de duración.

La fase de intervención se desarrolló a lo largo de 8 sesiones. Se trabajó con el niño durante cuatro días a la semana en sesiones de una hora de duración.

Los objetivos principales del programa de entrenamiento en funciones ejecutivas fueron cuatro:

- Mejorar la memoria de trabajo

- Reducir la velocidad de procesamiento

- Mejorar la conciencia fonológica

- Mejorar la competencia lectora inicial

Para conseguir en el estudiante un mayor nivel de autonomía cognitiva y comportamental, se aplicó la técnica de autoevaluación reforzada. Esta técnica pretendía potenciar el funcionamiento del sistema ejecutivo para que el niño lograse ser consciente de su propia conducta y supiera evaluarla en relación con las normas de clase.

El taller se inició con la actividad de "Normas y autoevaluación", en donde se discutió con el niño que normas son necesarias acordar y sus razones. Al finalizar 
cada sesión se dedican unos minutos para valorar que normas se han cumplido y cuáles no.

La intervención con el niño se realiza de una forma participativa, en la que se interactúa con el niño felicitándolo siempre cuando resuelve de forma adecuada el ejercicio y comprobando de forma metódica cual fue el error en aquellas que no consiguió resolver correctamente. Las actividades fueron diseñadas teniendo en cuenta los temas de interés del niño, tratando de finalizar cada sesión con actividades de tipo lúdico.

La última fase del trabajo consistió en la evaluación realizada al finalizar la intervención, la cual se llevó a cabo a lo largo de dos sesiones de hora y media de duración, empleando los mismos instrumentos que en la evaluación previa.

\section{Resultados}

A continuación se describen los resultados obtenidos en cada una de las pruebas empleadas en ambas fases de evaluación. En primer lugar se muestran los resultados obtenidos en la prueba LolEva (Fernández Amado et al., 2006; Mayor et al., 2011) con la cual se evaluó el nivel de conciencia fonológica y el nivel de competencia lectora inicial. Si se comparan los resultados de la prueba en su conjunto se pudo comprobar que se produjo una mejora, ya que en la evaluación previa a la intervención obtuvo una puntuación directa de 107, mientras que en la evaluación posterior la puntuación directa fue de 140 .

Analizadas por separado cada una de las subpruebas de conciencia fonológica, se pudo comprobar que en la subprueba de reconocimiento de rima, se produjo un pequeña incremento en la puntuación pasando de 8 en la evaluación inicial a 9 en la evaluación posterior (ver tabla 1). Aún así destaca el número elevado de aciertos en ambas aplicaciones puesto que el número de aciertos totales era de 10

Tabla 1

Comparación de los resultados obtenidos en la subprueba de Reconocimiento de rima en las dos fases de evaluación

\begin{tabular}{ccc}
\hline & Número de ítems & $\begin{array}{c}\text { Número de } \\
\text { aciertos }\end{array}$ \\
\hline $\begin{array}{c}\text { Previa a la } \\
\text { intervención } \\
\begin{array}{c}\text { Posterior a la } \\
\text { intervención }\end{array}\end{array}$ & 10 & 8 \\
\hline
\end{tabular}

Los resultados de las subpruebas que analizan la habilidad para segmentar la palabra en sílabas son también positivos, pues se produce una mejora en todas las subpruebas (ver tabla 2). Cabe señalar, que en la fase de evaluación posterior a la intervención, el niño consigue completar la subprueba de adición de sílaba inicial sin ningún error, obteniendo así la máxima puntuación de 5 .

También cabe destacar los resultados obtenidos en la subprueba de omisión de sílaba final ya que no obtuvo ningún acierto en la fase previa a la intervención, mientras que una vez finalizada la intervención consiguió 2 aciertos.
Tabla 2

Comparación de los resultados obtenidos en las subpruebas de sílaba en las dos fases de evaluación

\begin{tabular}{cccccccc}
\hline & \multicolumn{2}{c}{ Identificación } & \multicolumn{2}{c}{ Adición } & \multicolumn{2}{c}{ Omisión } \\
\hline & In & Fi & In & Fi & In & Fi \\
\hline $\begin{array}{c}\text { Previa a la } \\
\text { intervención } \\
\begin{array}{c}\text { Posterior a la } \\
\text { intervención }\end{array}\end{array}$ & 3 & 2 & 3 & 3 & 3 & 0 \\
\hline
\end{tabular}

Los resultados obtenidos en el conjunto de subpruebas que evalúan el nivel de dominio de los fonemas, se observó una mejora en todas a excepción de la subprueba de identificación del fonema inicial en la que no se produjeron cambios entre las dos fases de evaluación. Destacar que en la subprueba de adición de fonema final consiguió la máxima puntuación (5 aciertos) una vez finalizada la intervención.

Tabla 3

Comparación de los resultados obtenidos en las subpruebas de fonemas en las dos fases de evaluación

\begin{tabular}{cccccccc}
\hline & \multicolumn{2}{c}{ Identificación. } & \multicolumn{2}{c}{ Adición } & \multicolumn{2}{c}{ Omisión } \\
\hline & In & Fi & In & Fi & In & Fi \\
\hline $\begin{array}{c}\text { Previa a la } \\
\text { intervención } \\
\begin{array}{c}\text { Posterior a la } \\
\text { intervención }\end{array}\end{array}$ & 3 & 3 & 2 & 4 & 3 & 2 \\
\hline
\end{tabular}

En la figura 1 aparecen representado el porcentaje de aciertos obtenidos en las distintas subpruebas del LoLeva en las dos fases de evaluación.

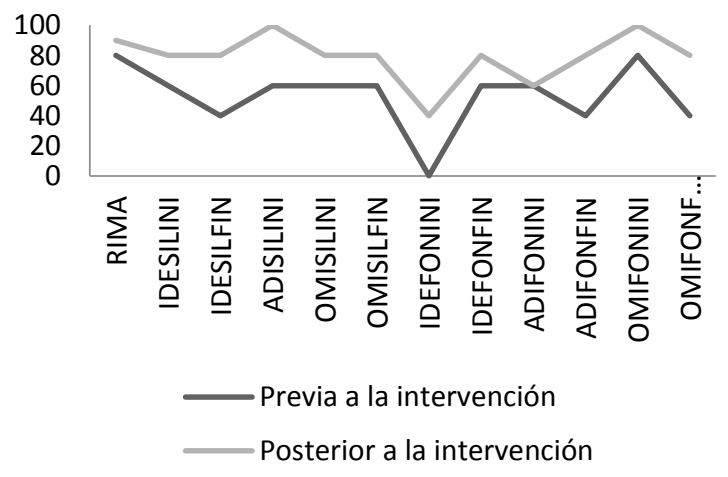

Figura 1. Comparación entre las dos fases de evaluación respecto al porcentaje de aciertos en las subpruebas de conciencia fonológica.

Respecto a los resultados obtenidos en las subpruebas que evalúan el nivel de competencia lectora inicial, indicar que en la lectura de letras se produjo una mejora tras el entrenamiento en funciones ejecutivas. Así, en la lectura de mayúsculas en la evaluación previa, el niño consiguió leer correctamente 20 letras de las 29 totales, mientras que en la evaluación posterior consiguió leer 24 . 
En el caso de la lectura de minúsculas también se produjo un pequeño incremento en el número de letras que es capaz de leer, ya que en la evaluación previa leyó correctamente 23 y en la evaluación posterior 25 .

Sin embargo, el tiempo empleado para la lectura de letras, tanto en el caso de las mayúsculas como en el de las minúsculas, se incrementó considerablemente tras la intervención (ver tabla 4).

Tabla 4

Número de aciertos y tiempo en la lectura de letras en las dos fases de evaluación

\begin{tabular}{cccccc}
\hline & \multicolumn{2}{c}{ Mayúsculas } & \multicolumn{2}{c}{ Minúsculas } \\
\hline & Aciertos & Tiempo & Aciertos & Tiempo \\
\hline $\begin{array}{c}\text { Previa a la } \\
\text { intervención }\end{array}$ & 20 & 49 & 23 & 43 \\
$\begin{array}{c}\text { Posterior a la } \\
\text { intervención }\end{array}$ & 24 & 94 & 25 & 67 \\
\hline
\end{tabular}

Los resultados en lectura de palabras también mostraron una mejoría, puesto que el número de aciertos es mayor en la evaluación posterior a la intervención (ver tabla 8). Teniendo en cuenta que el número máximo de aciertos era de 10 , en la evaluación realizada tras la intervención en lectura de palabras regulares, el niño consiguió realizarla sin cometer ningún error.

Si se compara el número de aciertos entre las distintas subpruebas, se observa un número similar de aciertos, tanto en la evaluación previa como posterior a la intervención. Esto es, en la lectura de palabras regulares, irregulares y pseudopalabras obtiene una puntuación de 7, 7 y 8 respectivamente, en la fase previa a la intervención, mientras que en la evaluación posterior a la intervención, las puntuaciones fueron de 10, 9 y 9 .

En cuanto al tiempo empleado para la lectura de palabras regulares, irregulares y pseudopalabras, al igual que en el caso de la lectura de letras, se ve incrementado en la evaluación posterior a la intervención.

Tabla 5

Número de aciertos y tiempo en la lectura de palabras en las dos fases de evaluación

\begin{tabular}{ccccccc}
\hline & \multicolumn{2}{c}{ Regulares } & \multicolumn{2}{c}{ Irregulares } & \multicolumn{3}{c}{ Pseudopalabras } \\
\hline & $\mathrm{A}$ & $\mathrm{T}$ & $\mathrm{A}$ & $\mathrm{T}$ & $\mathrm{A}$ & $\mathrm{T}$ \\
\hline $\begin{array}{c}\text { Previa a la } \\
\text { intervención }\end{array}$ & 7 & 33 & 7 & 28 & 8 & 27 \\
$\begin{array}{c}\text { Posterior a la } \\
\text { intervención }\end{array}$ & 10 & 70 & 9 & 61 & 9 & 71 \\
\hline
\end{tabular}

$\mathrm{A}=$ Aciertos, $\mathrm{T}=$ Tiempo

Al igual que en la lectura de letras y en la lectura de palabras, también se aprecia una leve mejoría en las puntuaciones obtenida en la subprueba de segmentación de palabras (ver tabla 6). La puntuación máxima en esta prueba era de 5 y consiguió una puntuación de 4 en la evaluación posterior a la intervención lo que significa que el niño muestra una adecuada habilidad.

Como en el resto de subpruebas de lectura el tiempo empleado es mayor en la evaluación posterior a la intervención.
Tabla 6

Número de aciertos y empleado en la segmentación de palabras en las dos fases de evaluación

\begin{tabular}{ccc}
\hline & Aciertos & Tiempo \\
\hline $\begin{array}{c}\text { Previa a la } \\
\text { intervención } \\
\begin{array}{c}\text { Posterior a la } \\
\text { intervención }\end{array}\end{array}$ & 3 & 43 \\
\hline
\end{tabular}

Como se puede observar en la figura 2 , se produce una mejora en todas las subpruebas que evalúan la competencia lectora inicial. El porcentaje de aciertos en el conjunto de las subpruebas es elevado ya que en ninguna es inferior al $50 \%$.

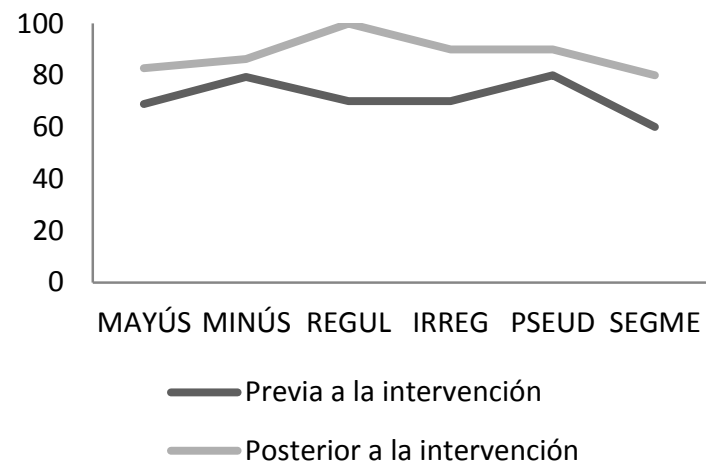

Figura 2. Comparación entre las dos fases de evaluación respecto al porcentaje de aciertos en las subpruebas de competencia lectora inicial.

En definitiva, los datos obtenidos en la prueba LolEva muestran una mejora tanto en la conciencia fonológica como en la competencia lectora inicial.

A continuación se muestran los resultados obtenidos en las dos pruebas empleadas del PEBL (Mueller y Piper, 2014): prueba Corsi y la prueba Letter-digit.

Los resultados obtenidos con la prueba de Corsi aparecen recogidos en la tabla 7, mostrando una incremento en amplitud de memoria que pasa de 3.5 a 4 .

Tabla 7

Resultados obtenidos en la prueba Corsi

\begin{tabular}{ccccc}
\hline & $\begin{array}{c}\text { Núm. } \\
\text { ensayo }\end{array}$ & Punt. & $\begin{array}{c}\text { Ensayos } \\
\text { correctos }\end{array}$ & $\begin{array}{c}\text { Span de } \\
\text { memoria }\end{array}$ \\
\hline $\begin{array}{c}\text { Previa a la } \\
\text { intervención }\end{array}$ & 9 & 20 & 5 & 3,50 \\
$\begin{array}{c}\text { Posterior a la } \\
\text { intervención }\end{array}$ & 11 & 30 & 6 & 4,00 \\
\hline
\end{tabular}

Las puntuaciones obtenidas en velocidad de procesamiento medida a través de la prueba Letter-digit se recogen en la tabla 8 . Como se observa en la fase previa a la intervención, la media del tiempo de respuesta fue de 5294,1 msg. mientras que en la fase posterior a la intervención obtuvo una puntuación media de 2351,2 msg. Por lo tanto, se produce una disminución en la velocidad de procesamiento tras la intervención. Cabe destacar que el número de aciertos es el máximo en ambas fases de evaluación. 
Tabla 8

Resultados obtenidos en la prueba Letter-digit

\begin{tabular}{ccc} 
& $\begin{array}{c}\text { Número de } \\
\text { aciertos }\end{array}$ & $\begin{array}{c}\text { Media tiempo de } \\
\text { reacción (msg) }\end{array}$ \\
\hline $\begin{array}{c}\text { Previa a la } \\
\text { intervención }\end{array}$ & 30 & 5294,1 \\
\hline $\begin{array}{c}\text { Posterior a la } \\
\text { intervención }\end{array}$ & 30 & 2351,2 \\
\hline
\end{tabular}

\section{Discusión y conclusiones}

Los resultados obtenidos tras llevar a cabo una intervención centrada en el desarrollo de las funciones ejecutivas, ponen de manifiesto que ésta influye de manera positiva en las cuatro variables objeto de estudio. Por lo tanto, estos resultados apuntan a la necesidad de incluir un entrenamiento de estas características como parte de la intervención en los TEA para mejorar principalmente conciencia fonológica y memoria de trabajo, lo que supondría una mejora en el proceso de adquisición lectora.

Sería recomendable que la duración de la intervención fuera mayor y comprobar en qué medida se continuaban produciendo las mejoras obtenidas. También sería conveniente hacer un seguimiento a largo plazo una vez finalizado el programa de intervención pues permitiría comprobar si las mejoras obtenidas se mantienen en el tiempo una vez finalizado el entrenamiento en funciones ejecutivas.

Para futuros estudios se recomienda ampliar la muestra y los grados de afectación con el fin de comprobar en qué medida es aconsejable este tipo de entrenamiento en niños diagnosticados de TEA con mayor afectación, cuanto influyen las funciones ejecutivas en la conciencia fonológica. Además podría ser recomendable llevar a cabo un estudio longitudinal que permitiera comprobar la evolución.

Además, futuros estudios deberían incluir alguna medida más acerca de otras funciones ejecutivas para comprobar si además de en la memoria de trabajo, el entrenamiento produce cambios en otras funciones .

\section{Referencias}

Artiles Hernández, C. y Jiménez González, J.E. (2006). Programa para la atención educativa al alumnado con TDAH. Consejería de Educación, Universidades, Cultura y Deportes del Gobierno de Canarias. Dirección General de Ordenación e Innovación Educativa.

Bravo Valdivieso, L. (2002). La conciencia fonológica como una zona de desarrollo próximo para el aprendizaje inicial de la lectura. Estudios pedagógicos, 28, 165-177.

Fernández-Amado, Mª. L., Mayor, M. A., Zubiauz, B., Tuñas, A. y Peralbo, M. (2006). Aplicación informática para la evaluación de la conciencia fonológica y competencia lectora. Comunicación presentada en el III Congreso Internacional de lectoescritura. AMEI-WAECE Asociación Mundial de
Educadores Infantiles. Morelia - México, 23 25 marzo de 2006.

Gabig, C. S. (2010). Phonological awareness and word recognition in reading by children with autism. Communication Disorders Quarterly, 31(2), 67-85.

Heimann, M., Nelson, K. E., Tjus, T., \& Gillberg, C. (1995). Increasing reading and communication skills in children with autism through an interactive multimedia computer program. Journal of autism and developmental disorders, 25(5), 459-480.

Mayor, Zubiauz, Peralbo Fernández-Amado, Tuñas y Brenlla, (2011). LOLE: From oral to written language. An instruction program for phonological awareness in kindergarten. Poster presentado en el 12th International Congress for the Study of Child Language. Montreal (Canadá), 19 al 23 de Julio.

Mueller, S.T. \& Piper, B.J. (2014). The Psychology Experiment Building Language (PEBL) and PEBL Test Battery. Journal of neuroscience methods 22, 250-259. doi: 10.1016/j.jneumeth.2013.10.024

Newman, T. M., Macomber, D., Naples, A. J., Babitz, T., Volkmar, F., \& Grigorenko, E. L. (2007). Hyperlexia in children with autism spectrum disorders. Journal of autism and developmental disorders, 37(4), 760-774.

Ozonoff, S., Pennington, B. F., \& Rogers, S. J. (1991). Executive function deficits in high-functioning autistic individuals: relationship to theory of mind.Journal of child Psychology and Psychiatry, 32(7), 1081-1105.

Rumsey, J. M. (1985). Conceptual problem-solving in highly verbal, nonretarded autistic men. Journal of autism and developmental disorders,15(1), 23-36.

Towgood, K. J., Meuwese, J. D., Gilbert, S. J., Turner, M. S., \& Burgess, P. W. (2009). Advantages of the multiple case series approach to the study of cognitive deficits in autism spectrum disorder. Neuropsychologia, 47(13), 2981-2988. 